\title{
SYSTEM PARAMETER ESTIMATION METHODS USING TEMPLATE FUNCTIONS
}

\author{
L. KEVICZKY and PHAM HUY THOA
}

\begin{abstract}
This paper presents a system parameter estimation method for correlated noise systems by using template functions and conjugate equations. The so-called extended template function estimator is developed on the basis of the conjugate equation theory. Under some weak conditions the parameter estimates obtained with the extended template function method are asymptotically Gaussian distributed. The covariance matrix of this distribution can then be used as a measure of the accuracy. In this paper it will be shown that this matrix can be optimized with respect to the vector of template functions and to the prefilter and that an optimal vector of template functions really do exist. With the optimal choice of the template function vector and of the prefilter, the proposed extended template function estimator reduces to the optimal instrumental variable estimator. When implementing the optimal template function method, a multistep algorithm consisting of four simple steps is proposed to estimate the system parameters and the parameters describing the noise characteristics.
\end{abstract}

Tóm tăt. Bài này trình bày một phương pháp đánh giá thông số hệ thống đối với các hệ ồn nhiễu có tương quan trên $c^{*}$ sờ các hàm mẫu và các phương trình liên hợp. Bộ đánh giá dùng hàm mẫu mở rộng được phát triển dựa trên lý thuyết các phương trình liên hợp. Trong một số điều kiện yếu, các đánh giá thông số nhận được bằng phương pháp hàm mẫu mở rộng có phân bố Gauss tiệm cận. Ma trận hiệp biến cưa phân bố này có thể được dùng như một thước đo độ chính xác. Trong bài báo này, chúng tôi sẽ chúng tỏ rặng ma trận này có thể được tối ư hóa đối với vectơ các hàm mẫu, đối với bộ tiền lọc và chúng minh sự tồn tại của vecto* tối u'u các hàm mẫu. Vói việc chọn tối ư vecto* các hàm mẫu và bộ tiền lọc, bộ đánh giá dùng các hàm mẫu mở rộng được đề xuất qui về bộ đánh giá biến dụng cụ tối ư. Khi thực hiện phương pháp hàm mẫu tối ưu, một thuật giải bao gồm bốn bước đơn giản đã được đề xuất để đánh giá các thông số hệ thống và các thông số mô tả các đặc trung của ồn nhiễu.

\section{INTRODUCTION}

A wide variety of system parameter estimation methods can be discussed from the point of view of functional operators working on system input/output signals. The classes of operators can be characterised by time functions, called template functions. Based on the notions of template functions [1], a multitude of system parameter estimation methods can be presented as a coherent picture. Template function based identification methods can be recognized as belonging to one of three related classes, with specific properties $[2,3,8]$. This leads to increased insight and to new, practical estimation schemes, adaptable for wide variety of situations.

Based on the theory of conjugate equations, the so-called extended template function estimator is developed in this paper. It will be shown that different system parameter estimators with specific properties can be obtained by particular choices of the prefilter and of the template functions. The vector of template functions and the prefilter can be chosen in many ways. They must fulfill the regularity conditions in order to give consistent parameter estimates. The choice of the template functions and of the prefilter will also influence the accuracy of the parameter estimates. The interesting question is how to choose the template function vector and the prefilter to achieve the best accuracy of the parameter estimates. There are different ways of expressing the accuracy. Under some weak conditions the parameter estimates obtained with the extended templattefunction piethod are asymptotically Gaussian distributed. The covariance matrix of this distribution can then be

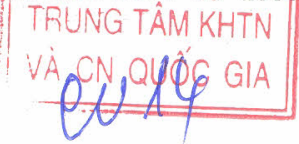


as a measure of the accuracy. In this paper it will be shown that this matrix can be optimized with respect to the vector of template functions and to the prefilter and that an optimal vector of template function really do exist. With the optimal choice of the template function vector and of the prefilter, the proposed extended template function estimator reduces to the optimal instrumental variable estimator presented in [6].

The optimal vector of template functions and the prefilter will, however, require the knowledge of the true system dynamics and also the statistical properties of the noise. To cope with this problem, a multistep algorithm consisting of four simple steps is then proposed when implementing the optimal template function method.

The paper is organized as follows. After preliminaries and some basic assumptions in Section 2, identification methods using template functions are briefly presented in Section 3. The so-called extended template function estimator is developed in Section 4 based on the theory of conjugate equations. The optimal template function estimator is derived in Section 5, where the optimation of accuracy is discussed. An iterative algorithm for estimating the noise parameters is given in Section 6. A multistep procedure is proposed in Section 7. Some conclusions are given in Section 8.

\section{PRELIMINARIES AND BASIC ASSUMPTIONS}

The system is assumed to be discrete-time, of finite order, and stochastic. It can be written as

$$
y(k)=\frac{B\left(q^{-1}\right)}{A\left(q^{-1}\right)} u(k-d)+v(k)
$$

where $y(k)$ is the output at time $k, u(k)$ is the input, $v(k)$ is a stochastic disturbance. Further, $q^{-1}$ is the backward time shift operator, $d$ is the discrete dead time, and

$$
\begin{aligned}
& A\left(q^{-1}\right)=1+a_{1} q^{-1}+a_{2} q^{-2}+\cdots+a_{n_{a}} q^{-n_{a}}, \\
& B\left(q^{-1}\right)=b_{0}+b_{1} q^{-1}+b_{2} q^{-2}+\cdots+b_{n_{b}} q^{-n_{b}} .
\end{aligned}
$$

The following standard assumptions on (2.1) will be made:

(A1) The polynomial $A(z)$, with $z$ being an arbitrary complex variable replacing $q^{-1}$, has all zeros outside the unit circle.

(A2) The polynomial $A(z)$ and $B(z)$ are coprime.

(A3) The input $u(k)$ is persistently exciting of order $n_{a}+n_{b}$, and is independent of the disturbance $v(k)$.

(A4) The disturbance $v(k)$ is assumed to be a stationary stochastic process with rational spectral density. It can be described as an ARMA process:

$$
v(k)=\frac{C\left(q^{-1}\right)}{D\left(q^{-1}\right)} w(k),
$$

where

$$
\begin{aligned}
& C\left(q^{-1}\right)=1+c_{1} q^{-1}+c_{2} q^{-2}+\cdots+c_{n_{c}} q^{-n_{c}}, \\
& D\left(q^{-1}\right)=1+d_{1} q^{-1}+d_{2} q^{-2}+\cdots+d_{n_{d}} q^{-n_{d}},
\end{aligned}
$$

and $w(k)$ is white noise with zero mean and variance $\lambda^{2}$.

The following assumption is added on (2.4):

(A5) The polynomials $C(z)$ and $D(z)$ are coprime.

If the degrees $n_{c}$ and $n_{d}$ are chosen to be unnecessary large, then this assumption is always fulfilled.

The overall system description then becomes

$$
y(k)=\frac{B\left(q^{-1}\right)}{A\left(q^{-1}\right)} u(k-d)+\frac{C\left(q^{-1}\right)}{D\left(q^{-1}\right)} w(k) .
$$

The system (2.5) can be written as 


$$
\begin{aligned}
A\left(q^{-1}\right) y(k) & =B\left(q^{-1}\right) u(k-d)+r(k), \\
r(k) & =H\left(q^{-1}\right) w(k)
\end{aligned}
$$

where $H\left(q^{-1}\right)$ is a finite order filter, $H\left(q^{-1}\right)$ as well as $H^{-1}\left(q^{-1}\right)$ are asymptotical stable

$$
H\left(q^{-1}\right)=\frac{A\left(q^{-1}\right) C\left(q^{-1}\right)}{D\left(q^{-1}\right)}
$$

For $k=1, \ldots, N$, the system equation (2.6) can be written in the vector/matrix form:

$$
\boldsymbol{A y}=\boldsymbol{B u}+\boldsymbol{r}
$$

where

$$
\begin{aligned}
\boldsymbol{r} & =\boldsymbol{H} \boldsymbol{w} \\
\boldsymbol{y} & =[y(1), \ldots, y(N)]^{T} \\
\boldsymbol{u} & =[u(1-d), \ldots, u(N-d)]^{T} \\
\boldsymbol{r} & =[r(1), \ldots, r(N)]^{T}
\end{aligned}
$$

and

$$
\begin{aligned}
& \boldsymbol{A}=\boldsymbol{I}+a_{1} \boldsymbol{S}_{N}^{1}+\cdots+a_{n_{a}} \boldsymbol{S}_{N}^{n_{a}}, \\
& \boldsymbol{B}=b_{0} \boldsymbol{I}+b_{1} \boldsymbol{S}_{N}^{1}+\cdots+b_{n_{b}} \boldsymbol{S}_{N}^{n_{b}} .
\end{aligned}
$$

Here, $\boldsymbol{S}_{N}^{(*)}$ denotes the TOEPLITZ shift matrix [4].

Denote the noise-free part of the output by $x(k)$, then

$$
A\left(q^{-1}\right) x(k)=B\left(q^{-1}\right) u(k-d) .
$$

Introduce the following vectors of delayed input and output values

$$
\begin{aligned}
& \varphi(k)=\left[-y(k-1), \ldots,-y\left(k-n_{a}\right), u(k-d-1), \ldots, u\left(k-d-n_{b}\right)\right]^{T}, \\
& \tilde{\varphi}(k)=\left[-x(k-1), \ldots,-x\left(k-n_{a}\right), u(k-d-1), \ldots, u\left(k-d-n_{b}\right)\right]^{T} .
\end{aligned}
$$

Introduce also the following parameter vectors, which describe the system transfer function as well as the noise correlation:

$$
\begin{aligned}
& \boldsymbol{\theta}^{*}=\left[a_{1}, \ldots, a_{n_{a}}, b_{0}, \ldots, b_{n_{b}}\right]^{T}, \\
& \boldsymbol{\beta}^{*}=\left[c_{1}, \ldots, c_{n_{c}}, d_{1}, \ldots, d_{n_{d}}\right]^{T} .
\end{aligned}
$$

Using the assumptions (A1) - (A3), it can be shown that

$$
\begin{aligned}
& E \boldsymbol{\varphi}(k) \boldsymbol{\varphi}^{T}(k) \geq E \tilde{\varphi}(k) \tilde{\varphi}^{T}(k)=E \tilde{\boldsymbol{\varphi}}(k) \boldsymbol{\varphi}^{T}(k)=E \boldsymbol{\varphi}(k) \tilde{\varphi}^{T}(k), \\
& E \tilde{\boldsymbol{\varphi}}(k) \tilde{\varphi}^{T}(k)>0,
\end{aligned}
$$

i.e., that the difference $E \varphi(k) \boldsymbol{\varphi}^{T}(k)-E \tilde{\varphi}(k) \tilde{\varphi}^{T}(k)$ is non-negative definite and the matrix $E \tilde{\varphi}(k) \tilde{\varphi}^{T}(k)$ is positive definite.

\section{TEMPLATE-FUNCTION-BASED IDENTIFICATION METHODS}

A wide variety of system parameter estimation methods can be discussed from the point of view of functional operators working on the system input/output signals. The classes of operators can be characterized by time functions, called template functions [1]. In the discrete-time case, these operators can be described by

$$
J[y(k)]=\langle p, y\rangle_{\Re^{N}}=\boldsymbol{p}^{T} \boldsymbol{y},
$$

where $p(k)$ is the template function and $\langle\cdot, \cdot\rangle_{\Re^{N}}$ denotes the inner product in $\Re^{N}$. 
For the system to be considered, it follows that

$$
J[y(k)]=J\left[\boldsymbol{\varphi}^{T}(k) \hat{\boldsymbol{\theta}}\right]+J[e(k)],
$$

where $e(k)$ is the equation error and $\hat{\boldsymbol{\theta}}$ is the estimate of $\boldsymbol{\theta}$.

Let us use $m$ operators (3.1) that are different in the sense that the corresponding template functions $p_{j}(k), j=1, \ldots, m$, are linearly independent, i.e., that they span an $m$-dimensional space

$$
j[\cdots]=\left[J_{1}[\cdots], \ldots, J_{m}[\cdots]\right]^{T} .
$$

If $m$ is chosen to be equal to $n_{\theta}=n_{a}+n_{b}+1$, i.e., there are many operators as there are parameters to be estimated, and if let the role of template functions $p_{j}(k)$ be played by $\boldsymbol{P}$, a matrix of the same dimension as $\psi$, then, along the line given before, we have

$$
\frac{1}{N} \boldsymbol{P}^{T} \boldsymbol{y}-\frac{1}{N} \boldsymbol{P}^{T} \boldsymbol{\psi}(\boldsymbol{y}, \boldsymbol{u}) \hat{\boldsymbol{\theta}}=\frac{1}{N} \boldsymbol{P}^{T} \boldsymbol{e}
$$

Here, $\boldsymbol{P}$ is called the template function matrix.

It is recognized that $\boldsymbol{e}$ is unobservable, and under certain conditions $\frac{1}{N} \boldsymbol{P}^{T} \boldsymbol{e}$ can be chosen to be equal to $\mathbf{0}[2]$. Then, it follows from Eq. (3.4) that

$$
\boldsymbol{P}^{T} \boldsymbol{y}=\boldsymbol{P}^{T} \boldsymbol{\psi}(\boldsymbol{y}, \boldsymbol{u}) \hat{\boldsymbol{\theta}}
$$

Consequently, the template function estimator can be written as

$$
\hat{\boldsymbol{\theta}}_{T F}=\left[\boldsymbol{P}^{T} \boldsymbol{\psi}(\boldsymbol{y}, \boldsymbol{u})\right]^{-1} \boldsymbol{P}^{T} \boldsymbol{y}
$$

provided of course that $\boldsymbol{P}^{T} \boldsymbol{\psi}$ is invertible.

Substitution of expression for the process output into Eq (3.6) leads to

$$
\hat{\boldsymbol{\theta}}_{T F}=\boldsymbol{\theta}^{*}+\left[\boldsymbol{P}^{T} \boldsymbol{\psi}(\boldsymbol{y}, \boldsymbol{u})\right]^{-1} \boldsymbol{P}^{T} \boldsymbol{r},
$$

from which statistical properties like (asymptotic) bias and (asymptotic) covariance can be found. From Eq. (3.6), we obtain different parameter estimators by making particular choices of the template function matrix $\boldsymbol{P}[2,3]$.

\section{THE EXTENDED TEMPLATE FUNCTION METHOD}

In this section, the so-called extended template function estimator will be developed on the basis of theory of conjugate equations $[7,8]$.

Consider now the system equation (2.6), which can be rewritten as

$$
H\left(q^{-1}\right) w(k)=A\left(q^{-1}\right) y(k)-B\left(q^{-1}\right) u(k-d) .
$$

For a moment, it is assumed that the filter $H\left(q^{-1}\right)$ is known a priopi, then the following estimation model corresponding to the system (4.1) can be used:

$$
\begin{gathered}
H\left(q^{-1}\right) \varepsilon(k)=\hat{A}\left(q^{-1}\right) y(k)-\hat{B}\left(q^{-1}\right) u(k-d), \\
\hat{y}(k)=\hat{\tilde{A}}\left(q^{-1}\right) y(k)-\hat{B}\left(q^{-1}\right) u(k-d)-\tilde{H}\left(q^{-1}\right) \varepsilon(k),
\end{gathered}
$$

where $\hat{\tilde{A}}\left(q^{-1}\right)=\hat{A}\left(q^{-1}\right)-1, \tilde{H}\left(q^{-1}\right)=H\left(q^{-1}\right)-1$ and $\varepsilon(k)$ is the prediction error

$$
\varepsilon(k)=y(k)-\hat{y}(k) .
$$

Let $F\left(q^{-1}\right)$ denote the prefilter of the input and output data. Then the estimation model can be extended as

$$
\begin{aligned}
F\left(q^{-1}\right) H\left(q^{-1}\right) \varepsilon(k) & =\hat{A}\left(q^{-1}\right) y^{F}(k)-\hat{B}\left(q^{-1}\right) u^{F}(k-d), \\
L\left(q^{-1}\right) \varepsilon(k) & =y^{F}(k)-\boldsymbol{\varphi}_{F}^{T}(k) \hat{\boldsymbol{\theta}}
\end{aligned}
$$


where

$$
\begin{aligned}
& L\left(q^{-1}\right)=F\left(q^{-1}\right) H\left(q^{-1}\right)=1+l_{1} q^{-1}+\cdots+l_{\infty} q^{-\infty}, \\
& y^{F}(k)=F\left(q^{-1}\right) y(k), u^{F}(k)=F\left(q^{-1}\right) u(k), \varphi_{F}^{T}(k)=F\left(q^{-1}\right) \varphi^{T}(k) .
\end{aligned}
$$

For $k=1, \ldots, N$, it can be presented in the matrix/vector form by

$$
\boldsymbol{L} \boldsymbol{\varepsilon}=\boldsymbol{y}^{F}-\boldsymbol{\psi}_{F}^{T} \hat{\boldsymbol{\theta}} .
$$

Corresponding to the functional operators $J_{p_{j}}[y(k)]$ working on the system output $y(k)$

$$
J_{p_{j}}=J_{p_{j}}[y(k)]=\left\langle p_{j}, y\right\rangle_{\Re^{N}}=\boldsymbol{p}_{j}^{T} \boldsymbol{y}=\sum_{k=1}^{N} p_{j}(k) y(k),
$$

the following operators $J_{p_{j}}[\hat{y}(k)]$ working on the model output $\hat{y}(k)$ are used:

$$
\hat{J}_{p_{j}}=J_{p_{j}}[\hat{y}(k)]=\left\langle p_{j}, \hat{y}\right\rangle_{\Re^{N}}=\boldsymbol{p}_{j}^{T} \hat{\boldsymbol{y}}=\sum_{k=1}^{N} p_{j}(k) \hat{y}(k),
$$

where $p_{j}(k), j=1, \ldots, m$, are the template functions and $\boldsymbol{p}(k)=\left[p_{1}(k), \ldots, p_{m}(k)\right]^{T}$ is called the vector of template functions.

In the matrix/vector form, the functional operators (4.4a) and (4.4b) can be described by

$$
\begin{aligned}
& \boldsymbol{j}=\left[J_{p_{1}}, \ldots, J_{p_{m}}\right]^{T}=\boldsymbol{P}^{T} \boldsymbol{y}, \\
& \hat{\boldsymbol{j}}=\left[\hat{J}_{p_{1}}, \ldots, \hat{J}_{p_{m}}\right]^{T}=\boldsymbol{P}^{T} \hat{\boldsymbol{y}},
\end{aligned}
$$

Introduce the conjugate equations corresponding to Eq. (4.3b):

$$
L^{*} \varphi_{j}^{*}(k)=g_{j}(k), \quad k=N, \ldots, 1, j=1, \ldots, m,
$$

where $L^{*}$ is the conjugate operator corresponding to $L\left(q^{-1}\right), g_{j}(k)$ are time functions, $\varphi_{j}^{*}(k)$ are called the conjugate functions and the vector of conjugate functions is denoted by $\varphi^{*}(k)=\left[\varphi_{1}^{*}(k), \ldots, \varphi_{m}^{*}(k)\right]^{T}$ The conjugate equations corresponding to Eq. (4.3c) are:

$$
\boldsymbol{L}^{*} \boldsymbol{\varphi}_{j}^{*}=\boldsymbol{g}_{j}, \quad j=1, \ldots, m
$$

or

$$
\boldsymbol{L}^{*} \phi^{*}=\boldsymbol{G},
$$

where $\boldsymbol{L}^{*}$ is the conjugate operator of $\boldsymbol{L}$ and $\boldsymbol{\phi}^{*}=\left[\boldsymbol{\varphi}_{1}^{*}, \ldots, \boldsymbol{\varphi}_{m}^{*}\right]$.

\section{Lemma 1.}

a) The conjugate operator for scalar polynomials is

$$
\operatorname{Conj}\left[P\left(q^{-1}\right)\right]=P\left(q^{-1} \hat{=} q\right)=P(q) .
$$

b) The conjugate operator for matrices is

$$
\operatorname{Conj}[\boldsymbol{P}(\boldsymbol{S})]=\boldsymbol{P}\left(\boldsymbol{S} \hat{=} \boldsymbol{S}^{T}\right)=\boldsymbol{P}\left(\boldsymbol{S}^{T}\right)=\boldsymbol{P}^{T} .
$$

Using Lemma 1, it follows from Eq. (4.5a) that

$$
L(q) \varphi_{j}^{*}(k)=g_{j}(k), \quad k=N, \ldots, 1, j=1, \ldots, m,
$$

where

$$
\begin{aligned}
& L(q)=1+l_{1} q+\cdots+l_{\infty} q^{\infty}, \quad \varphi_{j}^{*}(N+1)=\varphi_{j}^{*}(N+2)=\cdots=0, \\
& \boldsymbol{\varphi}^{*}(k)=\left[\varphi_{1}^{*}(k), \ldots, \varphi_{m}^{*}(k)\right]^{T},
\end{aligned}
$$

and from Eqs. $(4.5 \mathrm{~b})$ and $(4.5 \mathrm{c})$ that

$$
\boldsymbol{L}^{T} \boldsymbol{\varphi}_{j}^{*}=\boldsymbol{g}_{j},
$$




$$
\boldsymbol{L}^{T} \boldsymbol{\phi}^{*}=\boldsymbol{G}
$$

Theorem 1. Let $\varphi_{j}^{*}(k)$ be the solution of the conjugate equation (4.5a) with $g_{j}(k)=p_{j}(k)$ and $\delta J_{p_{j}}$ denote the variations of the functional operators given by Eq. (4.4). Then, the following relation holds

$$
\delta J_{p_{j}}=\sum_{k=1}^{N} p_{j}(k) \varepsilon(k)=\sum_{k=1}^{N} \varphi_{j}^{*}(k)\left[y^{F}(k)-\boldsymbol{\varphi}_{F}^{T}(k) \hat{\boldsymbol{\theta}}\right],
$$

where $\delta J_{p_{j}}=J_{p_{j}}-\hat{J}_{p_{j}}$.

The proof of Theorem 1 is given in the Appendix.

For $j=1, \ldots, m$, and $k=1, \ldots, N$, Eq. (4.8a) can be written in the form:

$$
\boldsymbol{\delta} \boldsymbol{j}=\boldsymbol{\phi}^{* T}\left[\boldsymbol{y}^{F}-\boldsymbol{\phi}_{F}(\boldsymbol{y}, \boldsymbol{u}) \hat{\boldsymbol{\theta}}\right]
$$

where $\boldsymbol{\delta} \boldsymbol{j}=\boldsymbol{j}-\hat{\boldsymbol{j}}$ and $\boldsymbol{\phi}^{*}=\left[\boldsymbol{\varphi}_{1}^{*}, \ldots, \boldsymbol{\varphi}_{m}^{*}\right]$.

As $\boldsymbol{\phi}^{*}$ is independent of $\hat{\boldsymbol{\theta}}$, the identification problem can be solved by using the following criterion:

$$
V=\boldsymbol{\delta} \boldsymbol{j}^{T} \boldsymbol{Q} \delta \boldsymbol{j}=\left\|\sum_{k=1}^{N} \boldsymbol{\varphi}^{*}(k)\left[y^{F}(k)-\boldsymbol{\varphi}_{F}^{T}(k) \hat{\boldsymbol{\theta}}\right]\right\|_{\boldsymbol{Q}}^{2} .
$$

Note that in (4.9) $\boldsymbol{Q}$ is a symmetric positive definite matrix.

Minimizing of the loss function $V$ results in

$$
\begin{gathered}
\hat{\boldsymbol{\theta}}=\left[\left(\sum_{k=1}^{N} \boldsymbol{\varphi}_{F}(k) \boldsymbol{\varphi}^{* T}(k)\right) \boldsymbol{Q}\left(\sum_{k=1}^{N} \boldsymbol{\varphi}^{*}(k) \boldsymbol{\varphi}_{F}^{T}(k)\right)\right]^{-1}\left[\left(\sum_{k=1}^{N} \boldsymbol{\varphi}_{F}(k) \boldsymbol{\varphi}^{* T}(k)\right) \boldsymbol{Q}\left(\sum_{k=1}^{N} \boldsymbol{\varphi}^{*}(k) y^{F}(k)\right)\right] \\
\hat{\boldsymbol{\theta}}=\left[\left(\boldsymbol{\psi}_{F}^{T}(\boldsymbol{y}, \boldsymbol{u}) \boldsymbol{\phi}^{*}\right) \boldsymbol{Q}\left(\boldsymbol{\phi}^{* T} \boldsymbol{\psi}_{F}(\boldsymbol{y}, \boldsymbol{u})\right)\right]^{-1}\left[\left(\boldsymbol{\boldsymbol { \varphi }}_{F}^{T}(\boldsymbol{y}, \boldsymbol{u}) \boldsymbol{\phi}^{*}\right) \boldsymbol{Q}\left(\boldsymbol{\phi}^{* T} \boldsymbol{y}\right)\right] .
\end{gathered}
$$

It should be stressed here that $\hat{\theta}$ is a consistent estimate of $\boldsymbol{\theta}^{*}$, i.e. $\hat{\boldsymbol{\theta}}$ converges with probability 1 (w.p.1) to $\theta^{*}$ as $N$ tends to infinity, if the matrix

$$
\lim _{N \rightarrow \infty} \frac{1}{N} \sum_{k=1}^{N} \boldsymbol{\varphi}^{*}(k) \boldsymbol{\varphi}_{F}^{T}(k)
$$

exists and is nosingular (w.p.1) and if

$$
\lim _{N \rightarrow \infty} \frac{1}{N} \sum_{k=1}^{N} \boldsymbol{\varphi}^{*}(k) r^{F}(k)=0 \quad \text { w.p.1, }
$$

where $r^{F}(k)=F\left(q^{-1}\right) r(k)$.

It is well-known that (4.11a) and (4.11b) are sufficient conditions for consistency. Under fairly general assumptions the limits and the summations in Eqs. (4.11a) and (4.11b) can be substituted with expectations [5]. Consequently the Eqs. (4.11a) and (4.11b) become

$$
\begin{aligned}
& E \varphi^{*}(k) \boldsymbol{\varphi}_{F}^{T}(k) \triangleq \boldsymbol{R} \text { has rank }\left(n_{a}+n_{b}+1\right), \\
& E \boldsymbol{\varphi}^{*}(k) r^{F}(k)=0 .
\end{aligned}
$$

The estimator given by Eq. (4.10) is called the extended template function estimator. By making particular choices of the template function vector $\boldsymbol{p}(k)$ and of the prefilter $F\left(q^{-1}\right)$ different estimators can be obtained [8]. Under the following various assumptions, the general estimator (4.10) reduces to somle well-known estimation schemes. 
i) With $m=\left(n_{a}+n_{b}+\dot{1}\right), F\left(q^{-1}\right)=1, \boldsymbol{p}(k)=H(q) \tilde{\boldsymbol{p}}(k), \tilde{\boldsymbol{p}}(k)$ being a template function vector, ( $\boldsymbol{Q}$ being irrelevant) it reduces to the basic template function method $[3,8]$.

ii) With $m=\left(n_{a}+n_{b}+1\right), F\left(q^{-1}\right)=1, \boldsymbol{p}(k)=H(q) z(k), z(k)$ being an vector of instruments, ( $\boldsymbol{Q}$ being irrelevant) it reduces to the ordinary instrumental variable method $[5,6,9]$.

iii) With $m=\left(n_{a}+n_{b}+1\right), F\left(q^{-1}\right)=H^{-1}\left(q^{-1}\right), \boldsymbol{p}(k)=z(k), z(k)$ being an vector of instruments, ( $\boldsymbol{Q}$ being irrelevant) it is an instrumental variable method with prefiltered data [9].

iv) With $\boldsymbol{Q}=\boldsymbol{I}, \boldsymbol{F}\left(q^{-1}\right)=H^{-1}\left(q^{-1}\right), \boldsymbol{p}(k)=H^{-1}\left(q^{-1}\right) \tilde{\varphi}(k)$, it is the optimal instrumental variable method $[5,6]$.

In the forthcoming analysis it is assumed that the vector of template functions $\boldsymbol{p}(k)$ can be chosen such that the vector of conjugate functions $\boldsymbol{\varphi}^{*}(k)$ is independent of the disturbance $r(t)$ for $k \geq t$. As the most common template functions used in practice are chosen to be linearly dependent on the input signal, the above assumption is trivially fulfilled. Note that due to this assumption the consistency condition (4.12b) is automatically satisfied, and that the matrix $\boldsymbol{R}$ in Eq. (4.12a) becomes

$$
\boldsymbol{R}=E \boldsymbol{\varphi}^{*}(k) \tilde{\varphi}_{F}^{T}(k)
$$

The results (4.12) and (4.13) will occasionally be referred in the following sections.

\section{THE OPTIMAL TEMPLATE FUNCTION ESTIMATOR}

The vector of template functions $\boldsymbol{p}(k)$ and the prefilter $F\left(q^{-1}\right)$ can be chosen in many ways. They must fulfill the regularity conditions given in (4.12) in order to give consistent parameter estimates. The choice of the template functions and of the prefilter will also influence the accuracy of the parameter estimates. The interesting question is how to choose the template function vector $\boldsymbol{p}(k)$ and the prefilter $F\left(q^{-1}\right)$ to achieve the best accuracy of the parameter estimates. There are different ways of expressing the accuracy. Under some weak conditions the parameter estimates obtained with the extended template function method are asymptotically Gaussian distributed. The covariance matrix of this distribution can then be used as a measure of the accuracy. In this section it will be shown that this matrix can be optimized with respect to the vector of template functions $\boldsymbol{p}(\kappa)$, to the prefilter $F\left(q^{-1}\right)$ and to the matrix $\boldsymbol{Q}$. The asymptotic distribution of the parameter estimates obtained is given in the following theorem.

Theorem 2. Consider the system described by Eqs. (2.1)-(2.4) and the extended template function estimator given by Eq.(4.10). Let $\varphi^{*}(k)$ be the vector of conjugate functions satisfying the conjugate equation. (4.7a) with $\boldsymbol{g}(k)=\boldsymbol{p}(k)$. Assume that (A1)-(A4) and (4.12)-(4.13) are satisfied. Then the estimate $\hat{\boldsymbol{\theta}}$ is asymptotically Gaussian distributed with

$$
\sqrt{N}\left(\hat{\boldsymbol{\theta}}-\boldsymbol{\theta}^{*}\right) / \lambda \stackrel{\text { dist }}{\longrightarrow} \mathcal{N}(0, \boldsymbol{P}),
$$

where $\boldsymbol{P}$ is the covariance matrix given by

$$
\begin{aligned}
\boldsymbol{P} & =\boldsymbol{P}(\boldsymbol{p}, F, \boldsymbol{Q}) \\
& =\left(\boldsymbol{R}^{T} \boldsymbol{Q R}\right)^{-1} \boldsymbol{R}^{T} \boldsymbol{Q}\left[E F\left(q^{-1}\right) H\left(q^{-1}\right) \boldsymbol{\varphi}^{*}(k) F\left(q^{-1}\right) H\left(q^{-1}\right) \boldsymbol{\varphi}^{* T}(k)\right] \boldsymbol{Q R}\left(\boldsymbol{R}^{T} \boldsymbol{Q R}\right)^{-1}
\end{aligned}
$$

and where $\boldsymbol{R}$ is defined in (4.13).

The theorem is proved following the method of proof of Theorem 4.1 in [6].

Next, it is interesting to find the optimal variables $\boldsymbol{p}^{\circ}(k), F^{\circ}\left(q^{-1}\right)$ and $\boldsymbol{Q}^{\circ}$ of the template function vector $\boldsymbol{p}(k)$, of the prefilter $F\left(q^{-1}\right)$ and of the matrix $\boldsymbol{Q}$ which give the maximum achievable accuracy. In other words, the variables $\boldsymbol{p}^{\circ}(k), F^{\circ}\left(q^{-1}\right)$ and $\boldsymbol{Q}^{\circ}$ have to be found such that

$$
\boldsymbol{P}\left(\boldsymbol{p}^{\circ}, F^{\circ}, \boldsymbol{Q}^{\circ}\right) \leq \boldsymbol{P}(\boldsymbol{p}, F, \boldsymbol{Q})
$$

for all $\boldsymbol{p}(k), F\left(q^{-1}\right)$ and $\boldsymbol{Q}$ fulfilling the required conditions. The relation (5.3) means that the difference $\boldsymbol{P}(\boldsymbol{p}, F, \boldsymbol{Q})-\boldsymbol{P}\left(\boldsymbol{p}^{\circ}, F^{\circ}, \boldsymbol{Q}^{\circ}\right)$ is nonnegative definite. 
This optimation problem can be solved by using the following theorem.

Theorem 3. Consider the covariance matrix $\boldsymbol{P}(\boldsymbol{p}, F, \boldsymbol{Q})$ given by (5.2). Assume that (A1) - (A4) and (4.12) are satisfied. Then

$$
\boldsymbol{P}(\boldsymbol{p}, F, \boldsymbol{Q}) \geq E\left[\boldsymbol{\beta}(k) \boldsymbol{\beta}^{T}(k)\right]^{-1},
$$

where $\boldsymbol{\beta}(k)=H^{-1}\left(q^{-1}\right) \tilde{\boldsymbol{\varphi}}(k)$ and the vector $\tilde{\boldsymbol{\varphi}}(k)$ is defined by Eq. (2.11). Moreover, equality in (5.4) holds if and only if $\boldsymbol{p}(k)=\left(\boldsymbol{R}^{T} \boldsymbol{Q}\right)^{-1} \boldsymbol{K} \boldsymbol{\beta}(k)$, where $\boldsymbol{K}$ is a constant and nonsingular matrix and $\boldsymbol{p}(k)$ denotes the vector of template functions defined in (4.4).

Proof. Note that the inverse in (5.4) exists since $A(z)$ and $B(z)$ are coprime and $u(k)$ is persistently exciting of order $\left(n_{a}+n_{b}+1\right)$, cf. (A2), (A3) and (2.14). Introduce the notation $\boldsymbol{\alpha}(k)=\boldsymbol{R}^{T} \boldsymbol{Q p}(k)$. Then it can be written

$$
\begin{aligned}
\boldsymbol{R}^{T} \boldsymbol{Q R} & =\boldsymbol{R}^{T} \boldsymbol{Q} E \boldsymbol{\varphi}^{*}(k) F\left(q^{-1}\right) \boldsymbol{\varphi}^{T}(k)=\boldsymbol{R}^{T} \boldsymbol{Q} E \boldsymbol{\varphi}^{*}(k) F\left(q^{-1}\right) \tilde{\boldsymbol{\varphi}}^{T}(k) \\
& =\boldsymbol{R}^{T} \boldsymbol{Q} E \boldsymbol{\varphi}^{*}(k) L\left(q^{-1}\right) H^{-1}\left(q^{-1}\right) \tilde{\boldsymbol{\varphi}}^{T}(k)=\boldsymbol{R}^{T} \boldsymbol{Q} E\left[L(q) \boldsymbol{\varphi}^{*}(k)\right]\left[H^{-1}\left(q^{-1}\right) \tilde{\boldsymbol{\varphi}}^{T}(k)\right] \\
& =\boldsymbol{R}^{T} \boldsymbol{Q} E \boldsymbol{p}(k) \boldsymbol{\beta}^{T}(k)=E \boldsymbol{\alpha}(k) \boldsymbol{\beta}^{T}(k)
\end{aligned}
$$

and

$$
\begin{aligned}
\boldsymbol{R}^{T} \boldsymbol{Q} & {\left[E F\left(q^{-1}\right) H\left(q^{-1}\right) \boldsymbol{\varphi}^{*}(k) F\left(q^{-1}\right) H\left(q^{-1}\right) \boldsymbol{\varphi}^{* T}(k)\right] \boldsymbol{Q R}=} \\
& =\boldsymbol{R}^{T} \boldsymbol{Q}\left[E L\left(q^{-1}\right) \boldsymbol{\varphi}^{*}(k) L\left(q^{-1}\right) \boldsymbol{\varphi}^{* T}(k)\right] \boldsymbol{Q R}=\boldsymbol{R}^{T} \boldsymbol{Q}\left\{E\left[\boldsymbol{\varphi}^{*}(k) L\left(q^{-1}\right)\right] L\left(q^{-1}\right) \boldsymbol{\varphi}^{* T}(k)\right\} \boldsymbol{Q R} \\
& =\boldsymbol{R}^{T} \boldsymbol{Q}\left[E\left(L(q) \boldsymbol{\varphi}^{*}(k)\right) L\left(q^{-1}\right) \boldsymbol{\varphi}^{* T}(k)\right] \boldsymbol{Q R}=\boldsymbol{R}^{T} \boldsymbol{Q}\left[E p(k) L\left(q^{-1}\right) \boldsymbol{\varphi}^{* T}(k)\right] \boldsymbol{Q R} \\
& =\boldsymbol{R}^{T} \boldsymbol{Q}\left[E\left(\boldsymbol{\varphi}^{*}(k) L\left(q^{-1}\right) \boldsymbol{p}^{T}(k)\right)^{T}\right] \boldsymbol{Q R}=\boldsymbol{R}^{T} \boldsymbol{Q}\left[E\left(L(q) \boldsymbol{\varphi}^{*}(k) \boldsymbol{p}^{T}(k)\right)^{T}\right] \boldsymbol{Q R} \\
& =\boldsymbol{R}^{T} \boldsymbol{Q}\left[E\left(\boldsymbol{p}(k) \boldsymbol{p}^{T}(k)\right)^{T}\right] \boldsymbol{Q R}=\boldsymbol{R}^{T} \boldsymbol{Q}\left[E \boldsymbol{p}(k) \boldsymbol{p}^{T}(k)\right] \boldsymbol{Q R}=E \boldsymbol{\alpha}(k) \boldsymbol{\alpha}^{T}(k) .
\end{aligned}
$$

Thus, Eq. (5.2) can be rewritten as

$$
\boldsymbol{P}(\boldsymbol{p}, F, \boldsymbol{Q})=\left[E \boldsymbol{\alpha}(k) \boldsymbol{\beta}^{T}(k)\right]^{-1}\left[E \boldsymbol{\alpha}(k) \boldsymbol{\alpha}^{T}(k)\right]\left[E \beta(k) \boldsymbol{\alpha}^{T}(k)\right]^{-1} .
$$

Since the matrix $\boldsymbol{Q}$ is assumed to be positive definite and $\boldsymbol{R}$ of full rank it follows that the matrix $\boldsymbol{P}$ given by Eq. (5.5) is positive definite. Therefore, the relation (5.4) implies

$$
E \beta(k) \boldsymbol{\beta}^{T}(k)-\left[E \beta(k) \boldsymbol{\alpha}^{T}(k)\right]\left[E \boldsymbol{\alpha}(k) \boldsymbol{\alpha}^{T}(k)\right]^{-1}\left[E \boldsymbol{\alpha}(k) \boldsymbol{\beta}^{T}(k)\right] \geq 0 .
$$

Since

$$
E\left[\begin{array}{c}
\boldsymbol{\beta}(k) \\
\boldsymbol{\alpha}(k)
\end{array}\right]\left[\boldsymbol{\beta}^{T}(k) \boldsymbol{\alpha}^{T}(k)\right] \geq 0,
$$

it follows easily that (5.6) is true.

If $\boldsymbol{\alpha}(k)=\boldsymbol{K} \boldsymbol{\beta}(k)$, with $\boldsymbol{K}$ nonsingular, then equality holds in (5.6). Conversely, if equality holds, then $\boldsymbol{\alpha}(k)=\boldsymbol{K} \boldsymbol{\beta}(k)$ with

$$
\boldsymbol{K}=\left[E \boldsymbol{\beta}(k) \boldsymbol{\alpha}^{T}(k)\right]\left[E \boldsymbol{\alpha}(k) \boldsymbol{\alpha}^{T}(k)\right]^{-1} .
$$

Replacing $\boldsymbol{\alpha}(k)=\boldsymbol{R}^{T} \boldsymbol{Q} \boldsymbol{p}(k)$ implies Theorem 3 has been proved.

It follows from Theorem 3 that with $\boldsymbol{K}=\boldsymbol{R}^{T} \boldsymbol{Q}$ the optimal vector of the template function to be found is

$$
\boldsymbol{p}^{\circ}(k)=H^{-1}\left(q^{-1}\right) \tilde{\boldsymbol{\varphi}}(k)
$$

This means in particular that the dimension of the template function vector is equal to the number of the system parameter to be estimated, i.e., $m=\left(n_{a}+n_{b}+1\right)$. Then the matrix $\boldsymbol{Q}$ does not influence the corresponding estimate (4.10). In the following it will be taken as the unit matrix $\boldsymbol{Q}^{\circ}=\boldsymbol{I}$.

With $\boldsymbol{Q}^{\circ}=\boldsymbol{I}$ and with $\boldsymbol{\varphi}^{*}(k)$ satisfying the required assumptions mentioned above, the extended 
template function estimator (4.10) reduces to

$$
\hat{\boldsymbol{\theta}}=\left[\sum_{k=1}^{N} \boldsymbol{\varphi}^{*}(k) \boldsymbol{\varphi}_{F}^{T}(k)\right]^{-1}\left[\sum_{k=1}^{N} \boldsymbol{\varphi}^{*}(k) y^{F}(k)\right] .
$$

Now, consider the conjugate equation (4.7a) with $\boldsymbol{g}(k)=\boldsymbol{p}^{\circ}(k)=H^{-1}\left(q^{-1}\right) \tilde{\varphi}(k)$. It can be rewritten as

$$
L(q) \varphi^{*}(k)=H^{-1}\left(q^{-1}\right) \tilde{\varphi}(k),
$$

where $L(q)=1+l_{1} q+\cdots+l_{\infty} q^{\infty}, \varphi^{*}(N+1)=\varphi^{*}(N+2)=\cdots=0, \varphi^{*}(k)=\left[\varphi_{1}^{*}(k), \ldots, \varphi_{m}^{*}(k)\right]^{T}$. This equation gives the condition for obtaining the lower bound (5.4) of the covariance matrix. It may have several solutions $\boldsymbol{\varphi}^{*}(k)$ depending on the prefilter $F\left(q^{-1}\right)$. Two convenient solutions are given in the following propositions.

Proposition 1. With the choice $F_{1}^{\circ}\left(q^{-1}\right)=H^{-1}\left(q^{-1}\right)$, the conjugate equation (5.11) does have the solution given by

$$
\boldsymbol{\varphi}_{1}^{* \circ}(k)=H^{-1}\left(q^{-1}\right) \tilde{\varphi}(k) .
$$

Proof. With $F_{1}^{\circ}\left(q^{-1}\right)=H^{-1}\left(q^{-1}\right)$, it follows that

$$
L_{1}\left(q^{-1}\right)=F_{1}^{\circ}\left(q^{-1}\right) H^{-1}\left(q^{-1}\right)=1 .
$$

According to Lemma 1 the conjugate operator is

$$
L_{1}(q)=1 .
$$

Thus, (5.12) is the solution of Eq. (5.11).

It is clear that with the prefilter $F_{1}^{\circ}\left(q^{-1}\right)$ and the solution $\boldsymbol{\varphi}_{1}^{* 0}(k)$, the consistency conditions (4.12) are satisfied and the matrix $\boldsymbol{R}$ defined in (4.13) is positive definite.

Corresponding to the optimal choice of the template function vector $\boldsymbol{p}^{\circ}(k)$ and the prefilter $F_{1}^{\circ}\left(q^{-1}\right)$, the system parameter estimate $(5.10)$ can be shown to be the following:

$$
\hat{\boldsymbol{\theta}}_{1}^{-}=\left[\sum_{k=1}^{N} H^{-1}\left(q^{-1}\right) \tilde{\boldsymbol{\varphi}}(k) H^{-1}\left(q^{-1}\right) \boldsymbol{\varphi}^{T}(k)\right]^{-1}\left[\sum_{k=1}^{N} H^{-1}\left(q^{-1}\right) \tilde{\varphi}(k) H^{-1}\left(q^{-1}\right) y(k)\right] .
$$

Proposition 2. With the choice $F_{2}^{\circ}\left(q^{-1}\right)=1$, the solution of the conjugate equation (5.11) is given by

$$
\boldsymbol{\varphi}_{2}^{* 0}(k)=H^{-1}(q) H^{-1}\left(q^{-1}\right) \tilde{\varphi}(k) .
$$

Proof. With $F_{2}^{\circ}\left(q^{-1}\right)=1$, the operator $L_{2}\left(q^{-1}\right)$ is

$$
L_{2}\left(q^{-1}\right)=F_{2}^{\circ}\left(q^{-1}\right) H\left(q^{-1}\right)=H\left(q^{-1}\right) .
$$

Using Lemma 1 the conjugate operator is found to be

$$
L_{2}(q)=H(q) .
$$

Thus, (5.14) is the solution of Eq. (5.11).

Corresponding to the optimal choice of $\boldsymbol{p}^{\circ}(k)$ and $F_{2}^{\circ}\left(q^{-1}\right)$, the following system parameter estimate is obtained from Eq. (5.10).

$$
\hat{\boldsymbol{\theta}}_{2}^{\circ}=\left[\sum_{k=1}^{N} H^{-1}(q) H^{-1}\left(q^{-1}\right) \tilde{\boldsymbol{\varphi}}(k) \boldsymbol{\varphi}^{T}(k)\right]^{-1}\left[\sum_{k=1}^{N} H^{-1}(q) H^{-1}\left(q^{-1}\right) \tilde{\varphi}(k) y(k)\right]
$$


Remark 1. The estimate (5.13) is identical with the optimal instrumental variable estimator proposed in $[5,6]$. The optimal instruments chosen by that method can be seen as the solution of the conjugate equation (5.11) with the optimal template function vector $\boldsymbol{p}^{\circ}(k)$ and the prefilter $F_{1}^{\circ}\left(q^{-1}\right)$.

Remark 2. Both the prefilter and the vector of template functions demand the knowledge of the true system parameters which are unknown a priori. Fortunately, it is possible to adaptively update these estimates as the estimation continues.

Remark 3. It is worth noting that there is no need to use additional template functions, i.e. to take the dimension of the template function vector larger than the number of the system parameters to be estimated, as far as optimal accuracy is concerned.

Remark 4. The first optimal estimate (5.13) relies heavily on the existence of a prefilter, while the second optimal estimate (5.15) does not require this. The computation of (5.15) requires both forward and backward filtering operations. However, the estimate (5.15) is not more involved computationally than (5.13).

\section{ESTIMATION OF THE NOISE PARAMETER VECTOR}

The parameter vector $\boldsymbol{\beta}^{*}$ of the ARMA noise model $C$ and $D$ given by Eq. (2.3) can be estimated by reference to $\hat{v}(k)$, the estimated value of the disturbance $v(k)$ in (2.1). Instead of (2.3) we will use the following ones:

$$
\boldsymbol{C w}=\boldsymbol{D} \hat{\boldsymbol{v}}
$$

where $\hat{\boldsymbol{v}}$ can be computed by

$$
\hat{\boldsymbol{v}}=\boldsymbol{y}-\hat{\boldsymbol{A}}^{-1} \hat{\boldsymbol{B}} \boldsymbol{u}
$$

Let us use the noise estimation model corresponding Eq. (4.22):

$$
\hat{C} \varepsilon=\hat{D} \hat{\boldsymbol{v}}
$$

where $\boldsymbol{E}$ is the prediction error.

As $\hat{\boldsymbol{v}}$ is a consistent estimate of the output error, a consistent estimate $\hat{\boldsymbol{\beta}}$ of the noise parameters $\boldsymbol{\beta}^{*}$ can also be obtained. The estimate $\hat{\boldsymbol{\beta}}$ can be found by applying the variational and conjugate equation methods presented in [7]. This method leads to the following iterative algorithm:

$$
\hat{\boldsymbol{\beta}}_{i+1}=\hat{\boldsymbol{\beta}}_{i}-\left[\boldsymbol{\psi}_{\hat{\boldsymbol{\theta}}, \hat{\boldsymbol{\beta}}_{i}}^{T}\left(\boldsymbol{\varepsilon}^{F}, \hat{\boldsymbol{v}}^{F}\right) \boldsymbol{\psi}_{\hat{\boldsymbol{\theta}}, \hat{\boldsymbol{\beta}}_{i}}\left(\boldsymbol{\varepsilon}^{F}, \hat{\boldsymbol{v}}^{F}\right)\right]^{-1} \boldsymbol{\psi}_{\hat{\boldsymbol{\theta}}, \hat{\boldsymbol{\beta}}_{i}}^{T}\left(\boldsymbol{\varepsilon}^{F}, \hat{\boldsymbol{v}}^{F}\right) \varepsilon_{\hat{\boldsymbol{o}}, \hat{\boldsymbol{\beta}}_{i}}
$$

where $\boldsymbol{\psi}_{\hat{\boldsymbol{o}}, \hat{\boldsymbol{\beta}}_{i}}\left(\boldsymbol{\varepsilon}^{F}, \hat{\boldsymbol{v}}^{F}\right)=\left[-\boldsymbol{S}_{N}^{1} \boldsymbol{\varepsilon}^{F}, \ldots,-\boldsymbol{S}_{N}^{n_{c}} \boldsymbol{\varepsilon}^{F}, \boldsymbol{S}_{N}^{1} \hat{\boldsymbol{v}}^{F}, \ldots, \boldsymbol{S}_{N}^{n_{d}} \hat{\boldsymbol{v}}^{F}\right]_{\hat{\boldsymbol{o}}, \hat{\boldsymbol{\beta}}_{i}}, \boldsymbol{\varepsilon}^{F}=\hat{\boldsymbol{C}}^{-1} \boldsymbol{\varepsilon}, \hat{\boldsymbol{v}}^{F}=\hat{\boldsymbol{C}}^{-1} \hat{\boldsymbol{v}}$

\section{A MULTisteP ALGORITHM}

On the basis of the results presented in the previous sections, a multistep algorithm for the parameter identification of the overall system (2.5) can be now proposed. It can be described simply in the following manner:

Step 1. The parameters of the polynomials $A$ and $B$ in the basic system model (2.1) are estimated using the solution (3.6). By choosing the template function matrix $\boldsymbol{P}$ a consistent estimate $\hat{\boldsymbol{\theta}}$ is obtained.

Step 2. Given the estimate $\hat{\boldsymbol{\theta}}$ from Step 1, an estimate $\hat{\boldsymbol{v}}$ of the noise $\boldsymbol{v}$ is computed as in Eq. (6.2) and the parameters of the ARMA noise model are estimated by reference to $\hat{\boldsymbol{\nu}}$ using the iterative algorithm (6.4). As the result a consistent estimate $\hat{\boldsymbol{\beta}}$ can also be obtained. 
Step 3. Compute the optimal template function vector (5.9) using $\hat{\boldsymbol{\theta}}$ from Step 1 and $\hat{\boldsymbol{\beta}}$ from Step 2 , solve the conjugate equation (5.11) with a particular choice of the prefilter and compute then the estimate of $\boldsymbol{\theta}^{*}$ by Eq. (5.10). As the result the optimal estimate $\hat{\boldsymbol{\theta}}_{\mathrm{OPT}}$ of $\boldsymbol{\theta}^{*}$ is obtained.

Step 4. Using, $\hat{\boldsymbol{\theta}}_{\mathrm{OPT}}$ from Step 3 in the computation of $\hat{\boldsymbol{v}}$ by Eq. (6.2), Step 2 can be repeated. It could be expected that the estimate $\hat{\boldsymbol{\beta}}$ in Step 4 will be more accurate than that of Step 2 .

Remark 5. An algorithm very closely related to the multistep procedure was presented in $[5,6]$. In that algorithm $\hat{\boldsymbol{\theta}}$ is computed by an arbitrary instrumental variable method in Step 1 and $\hat{\boldsymbol{\beta}}$ is determined in Step 2 by the extended matrix method or by the prediction error method. Step 3 in the multistep algorithm proposed above is more general than that given in $[5,6]$. They are identical only for a particular choice of the prefilter (see Proposition 1 and (5.13)).

\section{CONCLUSION}

In this paper we have developed a new parameter estimator for correlated noise systems by using template functions and conjugate equations. The so-called extended template function estimator has been developed on the basis of the conjugate equation theory. The parameter estimates obtained with such extended template function methods are proved to be Gaussian distributed under some weak conditions. The covariance matrix of this distribution is given explicitly and it used as measure of the accuracy. It has been shown that this matrix can be optimized with respect to the vector of template functions and to the prefilter and that an optimal vector of template functions really do exist. With the optimal choice of the template function vector and of the prefilter, the proposed extended template function estimator reduces to the optimal instrumental variable estimator. The optimal template function vector and prefilter obtained depend upon the unknown system dynamics and noise characteristics. Some approximate schemes must therefore be used when implementing the optimal template function method. A multistep algorithm consisting of four simple steps has been proposed to estimate the system parameters and the parameters describing the noise characteristics.

\section{APPENDIX}

Proof of Theorem 1

Multiplying both sides of $(4.3 \mathrm{~b})$ with $\varphi_{j}^{*}(k)$ and of $(4.7 \mathrm{a})$ with $\varepsilon(k)$, subtracting one from another and summing the final result from $k=1$ to $N$ one obtains from the left-hand side according to the definition of the conjugate operators

$$
\sum_{k=1}^{N} \varphi_{j}^{*}(k) L\left(q^{-1}\right) \varepsilon(k)-\sum_{k=1}^{N} L(q) \varphi_{j}^{*}(k) \varepsilon(k)=0
$$

and from the right-hand side

$$
\sum_{k=1}^{N} \varphi_{j}^{*}(k)\left[y^{F}(k)-\boldsymbol{\varphi}_{F}^{T}(k) \hat{\boldsymbol{\theta}}\right]-\sum_{k=1}^{N} g_{j}(k) \varepsilon(k)=\sum_{k=1}^{N} \varphi_{j}^{*}(k)\left[y^{F}(k)-\boldsymbol{\varphi}_{F}^{T}(k) \hat{\boldsymbol{\theta}}\right]-\sum_{k=1}^{N} p_{j}(k) \varepsilon(k) .
$$

It follows from (9.1), (9.2) and (4.4) that

$$
\delta \boldsymbol{J}_{p_{j}}-\sum_{k=1}^{N} \varphi_{j}^{*}(k)\left[y^{F}(k)-\boldsymbol{\varphi}_{F}^{T}(k) \hat{\boldsymbol{\theta}}\right]=0
$$

Eq. (9.3) implies Theorem 1 has been proved. 


\section{REFERENCES}

[1] Eykhoff P., System identification: approach to a coherent picture through template functions, Electronics Letters 16 (1980) 502-504.

[2] Eykhoff P., On the coherence among the multitude of system identification methods, Proc. 6th IFAC Sym. on Identification and System Parameter Estimation, Washington DC, 1982, 737-748.

[3] Eykhoff P., A. J. W. Van den Boom, and A. A. Van Rede, System identification methods - unification and information development using template functions, Proc. 8th IFAC World Congress, Kyoto, 1981.

[4] Keviczky L., C. Bányász, and M. Hilger, Folyamatidentifikáció, Folyamatirányítási Kutatások a Szilikátiparban, SZIKKTI, Budapest, 1977.

[5] Söderström T. and P. Stoica, Instrumental variable methods for identification of dynamical systems, Proc. IFAC Symp. on Identification and Parameter Estimation, York, UK, 1985, 17-28.

[6] Stoica P. and T. Söderström, Optimal instrumental variable estimation and approximate implementations, IEEE Trans. on AC 28 (7) (1983) 757-772.

[7] Thoa P. H., Identification techniques for multisensor systems, Proc. IFAC Symp. on Intelligent Components and Instruments for Control Applications, Budapest, Hungary, 1994, 156-160.

[8] Thoa P.H., Template functions based estimators for adaptive control, Proc. IFAC Sym. on Adaptive Systems in Control and Signal Processing, Budapest, Hungary, 1995, 250-255.

[9] Young P., The inştrumental variable method: a practical approach to identification and system parameter estimation, Proc. IFAC Symp. on Identification and Parameter Estimation, York, UK, 1985, 1-15.

Computer and Automation Research Institute,

Hungarian Academy of Sciences, Budapest, Hungary. 